\title{
Applications of Fiber-Optic Sensors
}

\author{
ALAN D. KERSEY AND ANTHONY DANDRIDGE
}

\begin{abstract}
There has been intense research in the area of fiber-optic sensors over the last decade. A wide range of application areas have been identified and the technology has now matured to the point where the sensor systems are commercially available from a number of companies. Furthermore, many other companies and research establishments are actively involved in the field-testing of prototype sensors systems.

This paper discusses the basic principles and applications of opticalfiber sensor technology, outlining the various sensing mechanisms which can be utilized to influence the propagation of light in an optical fiber and the various basic forms of sensor types-intensity, phase-modulated (inferometric), intrinsic, and extrinsic. Sensors for the measurement of temperature, pressure, liquid level, chemical parameters, acoustic, magnetic field, and rotation (gyroscope) are briefly described.
\end{abstract}

\section{INTRODUCTION}

$\mathrm{I}^{\mathrm{s}}$ N THE EARLY 1970's researchers studying multi-access single-fiber communications architectures were first to realize that light guided by an optical fiber could be modulated by direct physical perturbation and that this could be utilized in a variety of sensing applications [1]. Since this early work on intermodal modulation in multi-mode fibers, intensive research in a number of laboratories worldwide has resulted in the development of wide range of sensing techniques and configurations [2]-[5]. The diversity of optical phenomena, or "sensing mechanisms" employed in fiber sensors is quite incredible: for example, temperature can be measured by using no less than 18 fundamentally different methods using optical fibers. This allows sensors to be developed for different ranges and with different resolutions/accuracies for a manifold of application areas.

Fig. 1 shows the primary application areas for fiber sensor technology. The principle advantages of optical fiber sensors lie in their inherent safety due to electrically passive operation, and high immunity to electromagnetic interference (EMI) due to the dielectric nature of a fiber sensor system. In addition, optical sensing techniques in general offer a high sensitivity to a wide range of measurands with a wide dynamic range. Fiber-optic sensors provide geometric versatility and can be configured as "point" or "distributed" sensors. In this respect, fiber optics allow the development of unique sensor forms which can monitor a measurand in both the spatial and temporal domains-a technique for which there is not often an electrical/conventional counterpart. Where point sensing is of interest, combining fiber sensing with optical techniques

Manuscript received March 1, 1989; revised September 15, 1989. This paper was presented at the 39 th Electronic Components Conference, Houston, TX, May 22-24, 1989.

The authors are with the U.S. Naval Research Laboratory, Optical Sciences Division, Code 6574, Washington, DC 20375-5000.

IEEE Log Number 8933186

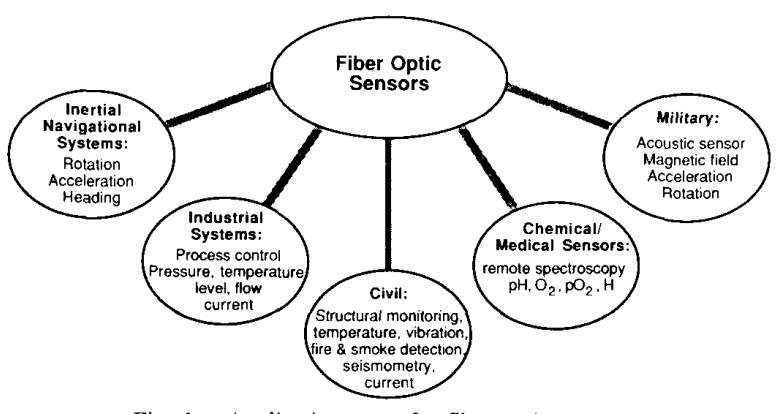

Fig. 1. Application areas for fiber-optic sensors.

to telemeter sensor data along passive fiber links allows the possibility of all-fiber sensor networks. This aspect of fiber sensor technology, which is currently receiving considerable research interest, may prove to be a major factor in determining the acceptance of fiber sensor technology in a range of application areas, especially in industrial process control type systems.

\section{SENSOR TYPES}

In general, fiber sensors can be classed as intensity-based or phase-modulated-based. The label "interferometric" is also commonly used to describe the latter class. Furthermore, there are two basic types of sensors; intrinsic-where the modulation of the optical signal occurs while the light is guided within the fiber, and extrinsic-where the light leaves the fiber, passes through some external transduction element, and is then recoupled back into a fiber. In the former case, the fiber itself is sensitized to the measureand field, whereas in the latter the external transduction element is responsive to the measurand. Fig. 2 shows the sensing mechanisms which can be described as intensity-based techniques. Here, the predominant approach has been to utilize some form of measurand induced attenuation in the sensing region. Other commonly used approaches involve spectral modulation, scattering, reflectance, and modal modulation. Techniques based on R.F subcarrier modulation of the optical source have also been developed. Typically, multimode fibers (i.e., 100 - to $600-\mu \mathrm{m} \mathrm{OD,} 25-$ to $500-\mu \mathrm{m}$ core $\varnothing$ ) are used in intensity-based sensor systems. Phase-modulated sensors operate by detecting measurand induced optical phase shifts between two or more coherent optical fields, and are generally based on fiber implementations of classical interferometers such as the Mach-Zehnder and Michelson configurations, and "differential" configurations where the interfering optical fields propagate in a common fiber (Fig. 3). Sensors based on hybrid systems, fiberlaunched laser Doppler velocimetry for example, have also

U.S. Government work not protected by U.S. Copyright 


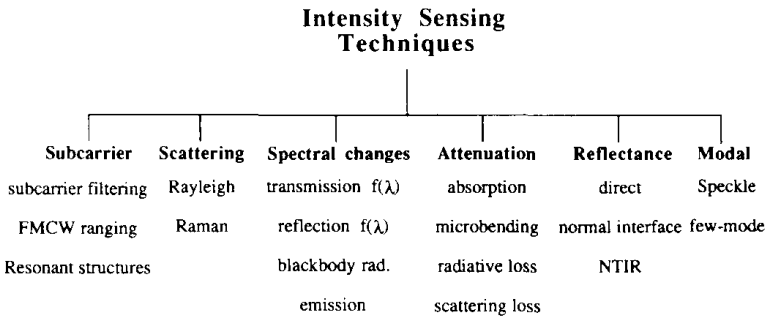

Fig. 2. Intensity-based sensing techniques.

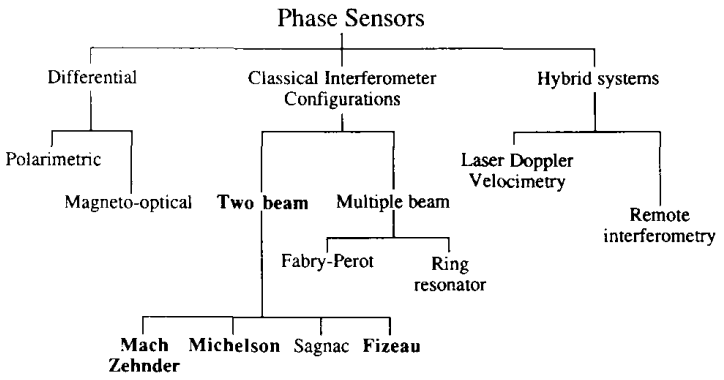

Fig. 3. Phase modulation based or interferometric sensors.

TABLE I

PRINCIPAL APPLICATION AREAS FOR FIBER-OPTIC SENSORS

\begin{tabular}{ll}
\hline Intensity-Based Sensors & Interferometric Sensors \\
\hline Temperature & Strain \\
Pressure & Pressure \\
Force & Acoustic fields \\
Strain & Magnetic fields \\
Torque & Electric fields \\
Flow & Rotation (gyroscope) \\
Liquid level & Acceleration \\
Position (displacement) & Vibration \\
Vibration & Velocity \\
Turbidity (oil pollution) & Temperature \\
Refractive index & Flow \\
Chemical & Current \\
Radiation & \\
Humidity & \\
\hline
\end{tabular}

received attention. Single-mode fibers (i.e., 80 - to $140-\mu \mathrm{m}$ $\mathrm{OD}, 4$ - to $10-\mu \mathrm{m}$ core $\varnothing$ ) are used in phase-modulated-based sensor systems. In this paper we will discuss primarily phasemodulated sensors based on the two-beam interferometer configurations, such as the Mach-Zehnder.

Table I lists the principal application areas for fiber-optic sensors. It can be seen that intensity-based sensors have been developed for primarily low-cost process-control/engineering type applications such as temperature, pressure, position, flow, liquid level, chemical sensing, etc., whereas with interferometric phase-modulated sensors more emphasis has been directed toward the development of high-performance sensors for inertial navigational systems, such as rotation, acceleration, and heading, and high-sensitivity devices for magnetic fields, acoustic fields, geophysical applications, etc.

\section{InTENSITy-Based Sensors}

Due to the diversity of intensity-based sensing techniques, it is not possible to present a complete overview of the tech- nology in this paper. We will, however, describe a number of different examples of sensor types designed for a variety of applications.

\section{Extrinsic Sensors}

A number of very simple, extrinsic, low-cost switch-type devices based on on/off modulation of an optical signal have been developed, and several have been engineered into commercial products. Fig. 4 shows three examples of this type of basic device for temperature, liquid level, and position. These devices operate on simple principles: the light is transmitted or reflected in an "on" state and blocked or not reflected in an "off" state. An array of fibers and an encoded plate form a basic position sensor. Clearly, this type of device has enormous potential for use in control systems such as in the aircraft industry, for example.

In most applications, however, an analog sensor is required. In general, in order to achieve a high measurement accuracy, this necessitates the use of a referencing scheme to avoid errors due to loss in connecting fibers, source drifts, etc. Commonly, a dual-wavelength approach is adopted in which light at one wavelength interacts with the transduction element, whereas the other bypasses the element or is not responsive to it; one wavelength then serves as the "signal" bearing optical carrier, the other as a reference. Fig. 5 shows an example of such a device [6]. Here, the dual-wavelength approach is used to sense temperature by monitoring the temperature-dependent color change in a thermochroic material encapsulated at the end of a fiber probe. The thermochroic effect is characterized by a temperature-dependent, spectrally selective absorption which gives rise to a reversible color change of the material with changing temperature. By using dual-wavelength optical interrogation of the probe, with one wavelength centered at the temperature-dependent absorption band, and the other well removed from it, a precise measurement of the transmissivity of the sample at the absorption wavelength can be made and the temperature of the sample determined. Similar principles have been used based on detecting absorption edge shifts in semiconductors [7], ruby glass [8], and rare-earth doped fibers [9].

The name "Optrode" has been coined to describe this type of extrinsic fiber sensor probe, especially when designed for use in medical, chemical, and biochemical applications [10]-[13], where the analogy to electrode is obvious. In one configuration used in chemical sensing, the measurand is detected by a probe material placed at the tip of the fiber, in a configuration similar to that shown in Fig. 5. Phenol red dye, which changes color as the $p \mathrm{H}$ of the surrounding material changes, was first used as a physiological $p \mathrm{H}$ sensor [10]. The optical absorbance of such a probe is dependent on $p \mathrm{H}$ for green light, but independent for red light. With the system illuminated by a white light source, the ratio of reflected green light to red light is thus $p \mathrm{H}$-dependent. This work demonstrated the detection of $p \mathrm{H}$ from 7.0 to 7.4 , with an accuracy of $0.01 \mathrm{pH}$ units. More recently, fiber-optic-based fluorometric techniques have been used to sense $p \mathrm{H}, \mathrm{PCO}_{2}$, $\mathrm{NH}_{3}$, lactate, and $\mathrm{O}_{2}$ [11], [12] to mention a few compounds. Sensitivities in the micromolar ranges have been achieved. In- 

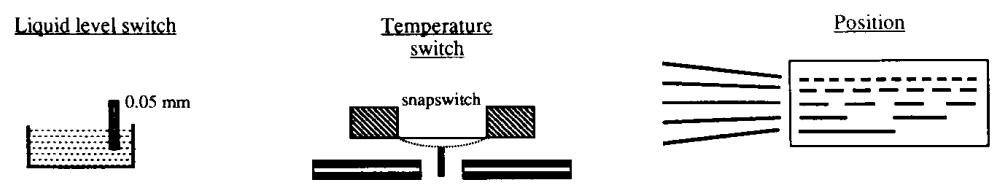

Fig. 4. Simple extrinsic intensity-based fiber-optic "switch" sensors.
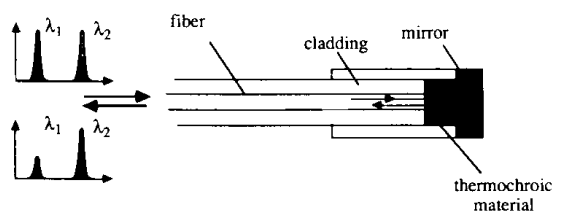

Fig. 5. Intensity-based temperature sensor utilizing differential absorption via the thermochroic effect.

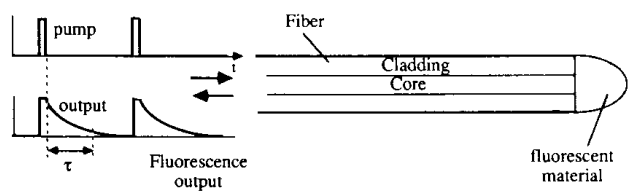

Fig. 6. Intensity-based extrinsic temperature sensor utilizing fluorescence decay time.

terest in this area is directed towards the development of sensor compatible with catheters for in vivo applications. Glucose and penicillin optrodes based on immobilized enzyme and indicator dyes at the fiber probe have also been developed [13].

A number of novel self-referenced intensity-based fiber sensor configurations have been reported in recent years. Fig. 6 shows an example of a device based on temperature-dependent fluorescence decay [14]. Here, the tip of a fiber is coated with a fluorescent material which is excited by an input optical pulse at a pump wavelength. Fluorescence signal in the material is captured by the fiber and guided back to a detector where the decay in the fluorescence signal is monitored. As the decay time is independent of the absolute optical power levels of the input pulse or returned fluorescence signal, the system is automatically self-referencing. Another example of such a device is the spectrum-modulating approach described recently for application in the measurement of temperature, pressure, and force. In this case, light from a broad-band source (wide spectral width) is coupled into a fiber input that guides the light to the sensing element which then returns a spectrally modulated optical signal. Changes in the measurand induce changes in the characteristics of the spectral modulation. There are a number of methods to implement this approach, however, the most commonly used involves the use of an external miniature Fabry-Perot cavity which is formed at the end of the fiber [15]-[17], as shown in Fig. 7. The cavity is formed by a partially reflective coating to the fiber end-face and a full reflector positioned in a substrate material responsive to a particular measurand, i.e., an elastic material in the case of pressure sensing. A measurand dependent change in the cavity length (between reflectors) results in a shift and change in periodicity in the spectral modulation of the returned optical signal. This approach appears particularly suitable for medical applications, such as in vivo monitoring

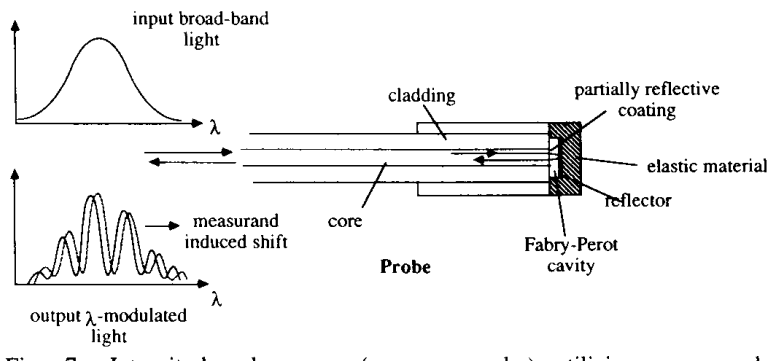

Fig. 7. Intensity-based sensor (pressure probe) utilizing measuranddependent spectral modulation.

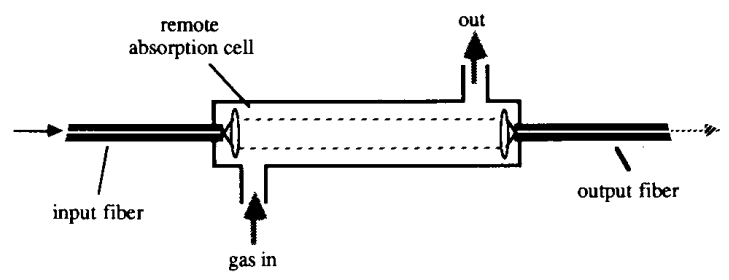

Fig. 8. Principle of fiber-optic remote spectroscopic detection of gases.

and temperature and pressure, where the small size, biocompatibility, and electrical passivity of fiber-optic sensors are of paramount importance.

In addition to the chemical/biochemical optrode-type sensors described above, extrinsic fiber sensors have also found application in a number of remote-spectroscopic-sensing applications. Probes for remote sensing of explosive gases have also been constructed in similar configurations to that shown in Fig. 8. These sensors typically use absorption spectroscopy to detect gases such as methane $\left(\mathrm{CH}_{4}\right)$, propane $\left(\mathrm{C}_{3} \mathrm{H}_{8}\right)$ and ethylene gas $\left(\mathrm{C}_{2} \mathrm{H}_{4}\right)$, etc., and utilize a longer absorption cell $(\sim 50 \mathrm{~cm})$ at the end of the fiber [18], [19]. Sensitivities of a few percent of the lower explosive limit (LEL) are readily achieved.

\section{Intrinsic Sensors}

In an intrinsic sensor, the fiber itself is responsive to the measurand field, and modulates the propagating light. Typically, this involves measurand-induced excess loss in the sensing fiber region, although measurand-dependent scattering, fluorescence, differential modal propagation, and optical length have all been utilized in various sensor systems reported to date.

Fig. 9 shows a schematic of an intrinsic sensor configuration based on microbending loss [20]. When a fiber is placed between the jaws of a deformer, the resulting microbending of the fiber causes light to be lost from the waveguide. This is a very sensitive technique, and large losses can be induced with relatively small deformation. The loss is not, however, a 


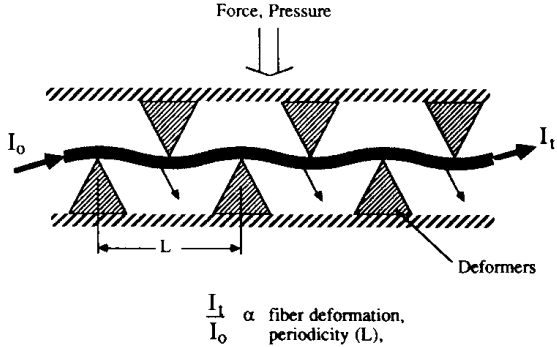

Fig. 9. Intrinsic "microbend" intensity-based fiber-optic sensor.

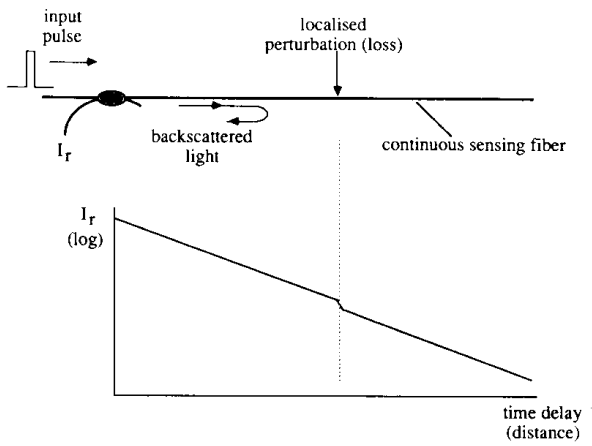

Fig. 10. Application of optical time-domain reflectometry (OTDR) to distributed sensing based on Rayleigh backscatter.

linear function of fiber deformation (as measured by the linear displacement of the laws), but exhibits a threshold in its transfer function characterized by a sharp increase in loss beyond a certain degree of microbending. Nevertheless, this can be used to some advantage for pressure/force-sensitive switches. The basic concept has been engineered to develop commercially available pressure pads and mats for use in industrial safety applications and automatically guided vehicles.

\section{Distributed Sensors}

By adopting certain signal processing techniques derived directly from free-space radar technologies, spatially different sections of a continuous sensing fiber can be separately interrogated, thus allowing the sensor to provide spatial as well as temporal variations in the measurand distribution. Intrinsic distributed sensors typically utilize either basic loss or scattering mechanisms in a single length of optical fiber which forms an extended sensor, the sensitivity of which may be enhanced to a particular measurand field. Interrogation of this extended sensor element using techniques such as optical time-domain reflectometry (OTDR) [21] permits the spatial variation of the measurand to be derived from the output information, thus allowing the measurand to be profiled within a certain spatial resolution along the fiber length. OTDR techniques are well established for fault/imperfection location and diagnostics in fiber communications applications. The most basic form of intrinsic-distributed sensor relies on the detection of regions of localized excess loss, due to-for example-microbending [22], in a length of fiber by direct OTDR analysis of the Rayleigh-backscattered light. The principle of operation is as shown in Fig. 10. In the case of measurand-dependent loss, a region of localized high loss due to perturbation of the fiber by the measurand field (stress, temperature, external refractive index, chemical interaction with a modified cladding, etc.) causes a change in the slope of the detected backscatter signal versus time-delay curve (OTDR curve) at a time delay corresponding to the spatial position of the perturbation. In principle, the returned power level at any instant could depend on various measurand-dependent parameters other than loss, such as scattering coefficient (elastic or nonelastic), fluorescence, stimulated emission, or the polarization properties of the light.

The first intrinsic distributed sensor concept was based on a variant of the conventional OTDR approach-polarizationoptical time-domain reflectometry (POTDR), in which the state of polarization of Rayleigh-backscattered light in a single fiber was detected as a function of time [23]. The first demonstration, however, of the use of measurand-dependent scattering as a means of distributed sensing utilized the change in the Rayleigh-scattering coefficient with temperature to perform distributed temperature sensing [24]. In general, this dependence is extremely weak in solid core fibers, and liquid core fibers were used to provide a higher sensitivity. A demonstration system, which exhibited $\sim 0.4 \%$ change in scattering coefficient per degree centigrade, showed that distributed sensing could be performed with good spatial ( $\sim$ few meters) and temperature $\left(\sim \pm 1^{\circ} \mathrm{C}\right)$ resolution. Various loss mechanisms can be utilized in distributed sensing, such as temperaturedependent absorption in rare-earth-doped fibers [9], temperature dependence of bending loss in plastic-clad (PCS) fibers [25], evanescent field radiative loss due to external refractive index changes [26] (due to for instance temperature variation in a fixed external medium, liquid leakage, or possibly chemically sensitized cladding), or continuous microbending loss in fiber. Distributed chemical sensing has also been proposed and demonstrated; for example, a distributed oxygen sensor based on fluorescence [27] was recently described.

Intrinsic distributed sensors are particularly attractive for use in applications where monitoring of a single measurand is required at a large number of points or continuously over the path of the fiber. Examples of application areas include stress monitoring in the evaluation of structural integrity of buildings, bridges, dams, storage tanks, etc., or mobile platforms (ships, oil platforms, aircraft, spacecraft, etc.), temperature profiling in electrical power transformers, generators, reactor systems, furnaces, process control systems, and simple fire detection systems. Distributed sensors can also be used for leakage detection in pipelines, distributed evanescentwave spectroscopy, fault diagnostics, and detection of magnetic/electrical field anomalies in power distribution systems, continuity testing of transportation networks, and to form simple intrusion alarm systems. Of particular interest in the area of intrinsic distributed sensing in the development of sensors embedded in composite materials for use in the real-time evaluation of stress, vibration, and temperature in structures and shells, especially in the aerospace industry.

Distributed temperature sensing using the temperature dependence of Raman scattering has also been demonstrated by several research groups [28]. The Raman scattering pro- 

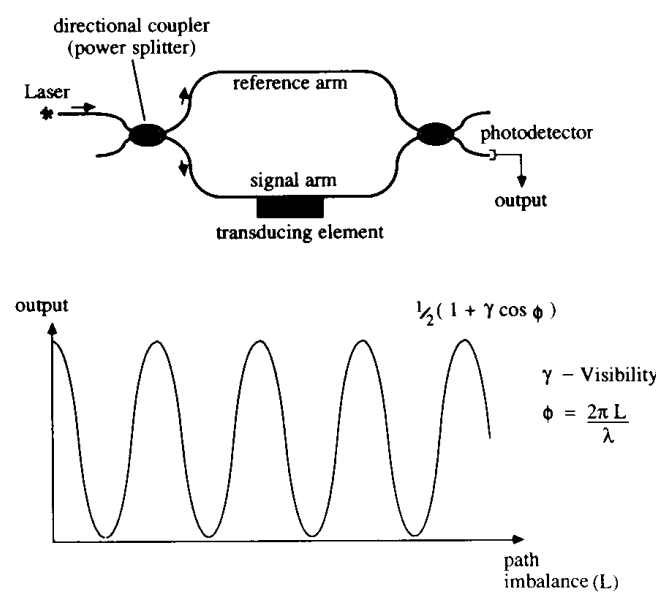

Fig. 11. Fiber-optic Mach-Zehnder interferometer phase-modulation-based sensor and output interference function.

cess produces components in a broad band about the exciting (pump) wavelength comprising Stokes (lower photon energy) and anti-Stokes (higher photon energy) emissions. The ratio of these intensities is temperature-dependent. Detection of the Raman backscatter typically involves the use of a modified OTDR technique in which the ratio of the Stokes to anti-Stokes levels of backscattered light is analyzed. Systems capable of operation over $\sim 1-\mathrm{km}$ fiber length with $\sim 1{ }^{\circ} \mathrm{C}$ resolution and 3- to $10-\mathrm{m}$ spatial resolutions have been demonstrated. At least two companies offer this instrument as a commercial product.

\section{Phase-Modulated Sensors}

Phase-modulated or interferometric fiber sensors offer the highest sensitivity to a wide range of measurands, including strain, pressure, acoustic field, acceleration (vibration), electric and magnetic fields, and temperature [2]. Interferometric sensor systems typically employ the Mach-Zehnder interferometer configuration shown in Fig. 11, although other configurations such as the Michelson and Fabry-Perot have also received some attention. In the Mach-Zehnder configuration, light from a single frequency (single longitudinal mode) laser, typically a diode laser, is coupled into a single-mode fiber and split into the two arms of the interferometer using a $2 \times 2$ fiber directional coupler (power splitter). One arm of the interferometer serves as the sensing arm, and is typically coated by or bonded to a measurand-responsive material (i.e., magnetostrictive material for magnetic field sensing) which strains the fiber. The other fiber arm serves as a reference path and is isolated from the measurand of interest. Light in the two arms is recombined using a second fiber coupler and interference between the two optical fields is detected using a photodetector. Measurand-induced changes in the length of the sensing fiber cause changes in the optical phase of the light from the sensing arm which results in a shift in the interference signal detected at the output. This approach is extremely sensitive to changes in optical path length; for time-varying modulations, the sensitivity of an interferometer is on the order of $\sim 10^{-13} \mathrm{~m}$ ( 1 part in $10^{14}$ for a $10-\mathrm{m}$ sensing fiber). The nonlinear "cosine-squared" dependence of the interference signal

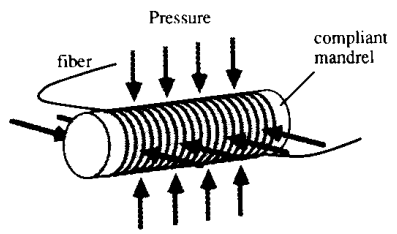

(a)

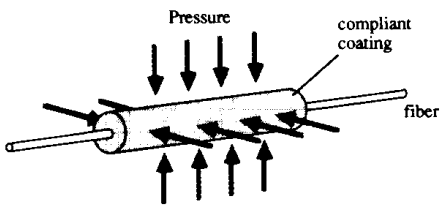

(b)

Fig. 12. Acoustic sensor configurations. (a) Mandrel sensor. (b) Coatedfiber sensor.

depicted in Fig. 10 can be linearized using a number of approaches based on passive homodyne or heterodyne-type signal processing schemes [29], [30] to provide wide dynamic range sensing $(>120 \mathrm{~dB})$.

As stated earlier, the fiber in the sensing arm of the interferometer is usually coated or bonded to a suitable transduction element which strains the optical fiber under the influence of the measurand field. The optical phase shift resulting due to this strain is due to two terms, namely

$$
\Delta \phi=\beta \Delta L+L \Delta \beta
$$

where $L$ is the length of the fiber, $\beta=2 \pi n / \lambda$ is the propagation constant, and $n$ is the refractive index of the core.

\section{Acoustic Sensor}

One of the major research areas of interferometric fiberoptic sensors is underwater acoustic sensing. The reasons for this interest ranges from cost, performance, geometric versatility of the sensing element, to networking capabilities. There are two main approaches used to couple an acoustic pressure into an optical phase shift in a fiber interferometer; the mandrel sensor and the coated-fiber sensor configurations (Fig. 12) [31]. In the mandrel sensor, fiber is wound under tension on a compliant mandrel. Compression of the mandrel under applied pressure is thus detected by a reduced fiber strain. With this design it is desirable to maximize the acoustic responsivity of the device, however, it is also important to achieve and maintain a flat acoustic response over the frequency range of interest for varying temperature and hydrostatic pressure levels. The majority of research work on interferometric fiber-optic acoustic sensors has used compliant plastic madrels-a typical example is nylon. The sensitivity is proportional to the reciprocal of the bulk modulus of the material, and for nylon a sensitivity in the range $5.5 \times 10^{-10}$ $\mathrm{rad} / \mathrm{m} \cdot \mu \mathrm{Pa}$ is achievable which varies by less than $5 \%$ over a 0 - to 1000 -psi pressure range and $0^{\circ} \mathrm{C}$ to $35^{\circ} \mathrm{C}$ temperature range. Sensors developed at NRL based on the plastic mandrel approach and using $\sim 30 \mathrm{~m}$ of sensing fiber have demonstrated below Sea State Zero (SSO) noise levels.

Whereas mandrel sensors appear ideal for use as "point" sensors, coated-fiber sensors have the advantage of extreme 


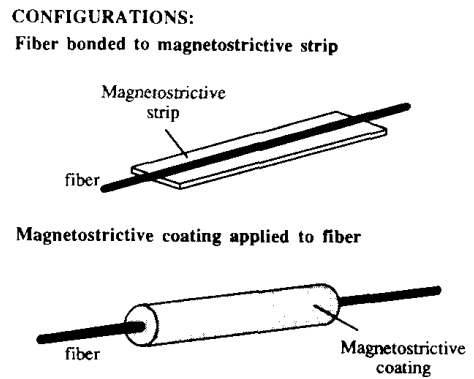

Fiber bonded to magnetostrictive cylinder

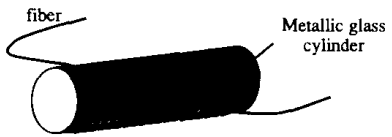

MATERIALS:

$$
\begin{aligned}
& \text { Nickel } \\
& \text { Iron-Nickel Alloys } \\
& \text { Coboit-Nickel Alloys } \\
& \text { Metallic Glasses }
\end{aligned}
$$

Fig. 13. Magnetic sensor configurations and materials.

geometric versatility. For example, a coated fiber of length 30 $\mathrm{m}$ could be wrapped to form a small golfball-sized omnidirectional sensor or used to form an extended element of 30 $\mathrm{m}$ in length. This type of approach usually leads to a lower acoustic scale factor $\{\Delta \phi /(L \cdot \Delta P)\}$, but as with the mandrel design, one which is relatively insensitive to temperature and pressure. Other areas of interest in fiber-optic acoustic sensing include the development of high-frequency sensors ( $>100 \mathrm{kHz}$ ) where sensor geometries and the use of composite structures play important roles in determining the sensor performance.

\section{Magnetic Sensor}

The main effort in recent research in the area of magnetic sensing using fiber interferometry has concentrated on techniques to increase the sensitivity for low-frequency magnetic field applications. Here, the preferred approach is to bond the fiber to a magnetostrictive material (Fig. 13), which under the influence of a magnetic field strains the fiber. Several materials have been investigated, ranging from nickel and nickel alloys to metallic glasses; metallic glasses appear to be close to the optimum material for this application exhibiting strong magnetostriction at low magnetic field strengths. Magnetostriction is a nonlinear effect, and in many materials the magnetostriction $(e)$ near zero magnetization is quadratic in nature, i.e.

$$
e=C H^{2}
$$

where $H$ is the applied magnetic field and $C$ is a constant depending on the material. The strain in the fiber is directly proportional to $e$. In order to make sensitive low-frequency measurements of magnetic fields using a fiber interferometer, the nonlinear behavior described by the above expression is utilized. By applying a magnetic "dither" field to the magne-

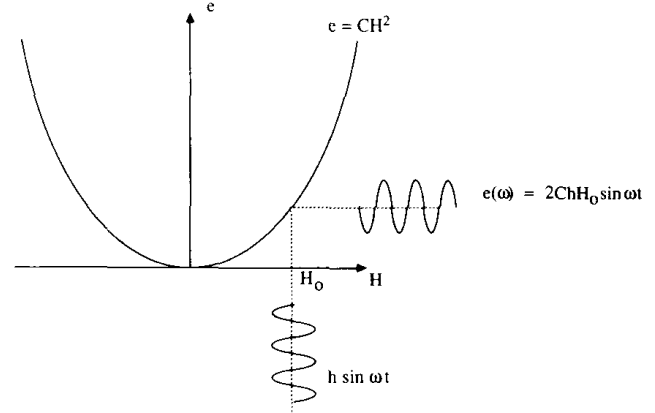

Fig. 14. Nonlinear nature of magnetostriction near zero magnetization of the sensing element.

tostrictive element, low-frequency magnetic field information can be up-converted to a higher frequency carrier where the measurement can be made under more favorable noise conditions, in particular away from $1 / f$ low-frequency phase noise and thermally induced phase fluctuations [32], [33]. As shown in Fig. 14, if the dither field is $h \sin \omega t$, and the dc ambient field is $H_{0}$, the strain in the fiber at the dither frequency can be shown to be given by

$$
\phi_{\omega} \alpha C h H_{0} \sin \omega t .
$$

For low-frequency magnetic field measurements, the amplitude of $\phi_{\omega}$ is directly proportional to the dc field $H_{0}$ and low-frequency variations in this field.

Sensitivities of $3 p T / \sqrt{ } \mathrm{Hz}$ at a frequency of $10 \mathrm{~Hz}$ have been reported recently for a fiber magnetometer based on this approach [34].

\section{Fiber-Optic Gyroscope (FOG)}

For over a decade, fiber interferometric techniques have been investigated for use in rotation sensing using the Sagnac effect [35]-[37]. Most interest has been in the use of the fiber ring or "Sagnac" interferometer configuration shown in Fig. 15 , although there is also considerable interest in the fiber ring resonator configuration. In the Sagnac interferometer, light from an optical source is split into two components which are coupled into a fiber loop of diameter $2 R$ and $N$ turns to form clockwise $(\mathrm{CW})$ and counter-clockwise $(\mathrm{CCW})$ propagating optical fields. After passing through the fiber loop, the two optical components are recombined in the coupler and the interference between them is detected at the output as shown. It should be noted that the output is derived from the same coupler port that is used as the input (via a second coupler (\#2)) and that a polarizer in the input to the fiber loop is used. This is required to ensure that the configuration is reciprocal; that is, the optical paths experienced by the $\mathrm{CW}$ and $\mathrm{CCW}$ optical field are identical in a nonrotating frame. In a rotating frame of reference (ohm degrees per second), the Sagnac effect causes the effective optical path through the loop to increase for one beam, while for the other it becomes shortened. This results in a phase shift

$$
2 \phi_{s}=\frac{8 \pi N A}{\lambda c} \Omega
$$




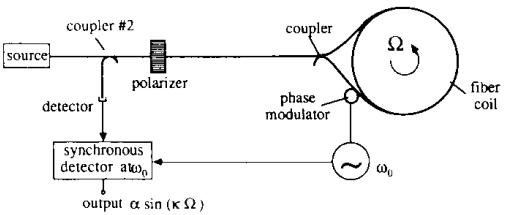

Fig. 15. Fiber-optic gyroscope based on the "open-loop" Sagnac interferometer configuration.

between the two optical components at the output and thus a shift in the output interference signal. In the above expression, $A=\pi R^{2}$ is the area of coil, $\lambda$ is the source wavelength, and $c$ is the velocity of light. As an example of the magnitude of this effect, a 1000 -turn fiber coil of $20-\mathrm{cm}$ diameter rotating at $1^{\circ} / \mathrm{h}$ produces a phase shift of approximately $10^{-5} \mathrm{rad}$ (assuming $\lambda \sim 1 \mu \mathrm{m}$ ). A considerable amount of effort has been expended over the last decade on the development of signal processing techniques to derive a linear output with rotation. Details of that research are beyond the scope of this paper, and the interested reader is referred to some recent reviews on the subject [36], [37].

Bias drift rates of $\sim 0.005^{\circ} / \mathrm{h}$ have been demonstrated with the all-fiber gyroscope-close to that required for many inertial navigational systems. Another important performance requirement is that of scale factor stability. This requirement is application-dependent, and can range from $\sim 1 \%$ for lowcost systems to $\sim 0.001 \%$ for long-term platform stabilization and other high-performance systems.

\section{Other Applications}

The application areas for fiber-optic interferometric sensors range from high-performance inertial navigation, military and civil systems, to industrial process control type systems. As the interferometer is essentially a highly sensitive strain gauge it is not surprising that strain measurements were one of the first applications of the technology [38]. Highly sensitive accelerometers can be formed using conventional massspring techniques, and deformable acceleration-sensitive mandrels [39], [40]. This leads to applications in seismometry and vibration analysis [41]. As indicated earlier, fiber interferometers can be configured to measure pressure and temperature [42] with high resolution, and just as magnetostrictive materials can be employed in magnetic sensors, electric fields can be detected using piezoelectric fiber coatings or elements [43]. Fiber interferometry has also been developed for use in the measurement of liquid flow rate sensors [44], [45].

\section{SUMMARY}

We have discussed the principle of operation and application areas of a range of intensity-based and phase-modulationbased fiber-optic sensor systems. A large number of sensor types have been demonstrated over the past 15 years, and the technology has now matured to the point where a number of companies offer fiber sensors as commercial products. Much of the current development effort is being devoted to the engineering and packaging of tried-and-tested sensing techniques to produce reliable, ruggedized sensor systems which can capitalize on the performance advantage that this technology can offer.
REFERENCES

[1] D. E. N. Davies and S. Kingsley, Electron. Lett., vol. 10, p. 21, 1974.

[2] T. G. Giallorenzi et al., IEEE J. Quantum Electron., vol. QE-18, p. $626,1982$.

[3] B. Culshaw, Optical Fibre Sensing and Signal Processing. London, UK: Peter Peregrinus, 1984.

[4] G. D. Pitt et al., Proc. Inst. Elec. Eng., pt. J, vol. 132, p. 214, 1985.

[5] See for example: Proc. 5th Int. Conf. on Optical Fiber Sensors (OFS'88) (New Orleans, LA, Jan. 1988), published by OSA.

[6] M. Brenci et al., in Proc. OFS' 84 (Stuttgart, Germany, 1984), p. 155.

[7] D. A. Christensen and V. A. Vaguine, Proc. SPIE, vol. 838 , p. 252 , 1987.

[8] E. Theocharous, in Proc. 1st Int. Conf. on Optical Fiber Sensors, (IEE, London), p. 10, 1983

[9] D. N. Payne, in Proc. Int. Conf. on Optical Fiber Communications (OFC'87) (Reno, NV, 1987), p. 166.

[10] J. I. Peterson and G. G. Vurek, "Fiber optic sensors for biomedical applications," Science, vol. 224, p. 123, 1984.

[11] R. Seitz, "Chemical sensors based on optical fibers," Anal. Chem., vol. 66, 1984.

[12] J. F. Giuliani et al., "Reversible optical waveguide sensor for ammonia vapors," Opt. Lett., vol. 8, p. 54, 1983.

[13] A. M. Scheggi and G. Conforti, "Review: Optical fiber sensors and applications with particular reference to chemical sensing," in Proc. Fibre Optics ' 88 (London, UK, Apr. 1988), (SIRA/SPIE Pub., vol. 949).

[14] Th. Bosselman, in Proc. 2nd OFS (Stuttgart, Germany, 1984), p. 151.

[15] E. R. Cox and B. E. Jones, in Proc. 1st Int. Conf. on Optical Fiber Sensors (IEE, London), p. 122, 1983.

[16] R. A. Lieberman et al., in Proc. OFC' 87 (Reno, NV, 1987), p. 75.

[17] G. Beheim et al., Proc. SPIE, vol. 838, p. 238, 1987; also J. C. Hartl et al., p. 257.

[18] S. Stueflotten et al., "An infrared fiber optic gas detection system," in Proc. OFS'84 (Stuttgart, Germany, Sept. 1984), p. 87.

[19] K. Chan et al., "All optic fiber based remote sensing system for nearinfrared absorption of low-level $\mathrm{CH}_{4}$ gas," J. Lightwave Technol., vol. LT-5, p. 1706, 1987.

[20] G. F. Lipscomb et al., in Proc. Ist Int. Conf. on Optical Fiber Sensors (IEE, London), p. 117, 1983.

[21] A. D. Kersey and A. Dandridge, Proc. Inst. Electronic Radio Eng. (IERE, London), vol. 58, p. S99, 1988.

[22] A. R. Mickelson et al., J. Lightwave Technol., vol. LT-2, p. 700 , 1984.

[23] A. J. Rogers, Appl. Opt., vol. 20, p. 1060, 1981

[24] A. H. Hartog, J. Lightwave Technol., vol. LT-1, p. 498, 1983

[25] P. Heinzman and R. Hofstetter, Proc. SPIE, vol. 584, 1985.

[26] P. M. Kopera et al., Proc. SPIE, vol. 412, p. 82, 1983.

[27] R. A. Lieberman et al., "Distributed fluorescence oxygen sensor," in Proc. OFS' 88 (New Orleans, LA, Jan. 1988), p. 346.

[28] J. P. Dakin et al., Electronic Lett., vol. 21, p. 569, 1985.

[29] A. Dandridge et al., IEEE J. Quantum Electron., vol. QE-18, p. 1647, 1982 .

[30] A. D. Kersey et al., in Proc. Ist Int. Conf. on Optical Fiber Sensors (IEE, London), p. 43, 1983.

[31] J. A. Bucaro et al., "Fiber optic acoustic sensors," in Physical Acoustics XVI. New York, NY: Academic Press, 1982.

[32] K. P. Koo and G. H. Sigel Jr., J. Lightwave Technol., vol. LT-1, p. $509,1983$.

[33] A. D. Kersey et al., Electron. Lett., vol. 20, p. 573, 1984.

[34] D. Dagenais et al., Appl. Phys. Lett., vol. 53, p. 1474, 1988

[35] V. Vali and R. W. Shorthill, Appl. Opt., vol. 15, p. 1099.

[36] B. Culshaw and I. P. Giles, J. Phys. E. Sci. Instrum., vol. 16, p. 5 , 1983.

[37] See: Proc, SPIE, vol. 719, Fiber Optic Gyros, E. Udd, Ed. Cambridge MA, 1986

[38] C. D. Butler and G. B. Hocker, Appl. Opt., vol. 17, p. 2867, 1978.

[39] A. B. Tveten et al., Electron. Lett., vol. 16, p. 854, 1980.

[40] A. D. Kersey et al., Electron. Lett., vol. 18, p. 559, 1982

[41] C. M. Davis et al., Proc. SPIE, vol. 718, p. 203, 1986.

[42] M. Corke et al., Electron. Lett., vol. 19, p. 471, 1983.

[43] K. P. Koo and G. H. Sigel, IEEE J. Quantum Electron., vol. QE-18, p. $670,1982$.

[44] P. Akhavan Leilabady et al., in Proc. 2nd Int. Conf. on Optical Fiber Sensors (Stuttgart, Germany, 1984), p. 35.

[45] C. A. Wade and A. Dandridge, Electron. Lett., vol. 24, p. 785, 1988 